\title{
Long term seismic noise acquisition and analysis in the Homestake mine with tunable monolithic sensors
}

\author{
Fausto Acernese ${ }^{a, b}$, Rosario De Rosa ${ }^{b, c}$, Riccardo De Salvo ${ }^{d}$, Gerardo Giordano $^{a}$, Jan Harms ${ }^{e}$, \\ Vuk Mandic $^{e}$, Angelo Sajeva ${ }^{e}$, Thomas Trancynger ${ }^{f}$, Fabrizio Barone ${ }^{a, b}$ \\ ${ }^{a}$ Università degli Studi di Salerno, Fisciano (SA), Italia \\ ${ }^{b}$ INFN sez. di Napoli, Napoli, Italia \\ ${ }^{c}$ Università di Napoli "Federico II", Napoli, Italia \\ ${ }^{d}$ California Institute of Technology, Pasadena, CA, U.S.A. \\ ${ }^{e}$ University of Minnesota, Twin Cities, MN, U.S.A. \\ $d$ Università di Pisa, Pisa, Italia \\ ${ }^{f}$ South Dakota Science and Technology Authority, Lead, SD, U.S.A.
}

\begin{abstract}
In this paper we describe the scientific data recorded along one month of data taking of two mechanical monolithic horizontal sensor prototypes located in a blind-ended (side) tunnel $2000 \mathrm{ft}$ deep in the Homestake (South Dakota, USA) mine chosen to host the Deep Underground Science and Engineering Laboratory (DUSEL). The two mechanical monolithic sensors, developed at the University of Salerno, are placed, in thermally insulating enclosures, onto concrete slabs connected to the bedrock, and behind a sound-proofing wall. The main goal of this experiment is to characterize the Homestake site in the frequency band $10^{-4} \div 30 \mathrm{~Hz}$ and to estimate the level of Newtonian noise, providing also the necessary preliminary information to understand the feasibility of underground gravitational-wave interferometers sensitive at $1 \mathrm{~Hz}$ and below.
\end{abstract}

Keywords: Monolithic Sensor, Folded Pendulum, Seismometer, Seismic Noise, Gravitational Waves

\section{INTRODUCTION}

The Homestake mine became famous as the largest known iron-formation-hosted gold-ore body in the world and is considered as the prototype of its kind. It is situated in the Lead-Deadwood Dome of the Black Hills which is marked by a rather complicated stratification and history of folding events and metamorphosis of igneous and sedimentary rocks. Two uplifts have brought the Black Hills into its present configuration.

In January 2002, the Barrick Gold Corporation ceased operation of the mine which was then donated to the state of South Dakota. It is being transformed gradually into a scientific laboratory controlled by the South Dakota Science and Technology Authority (SDSTA). The endeavor is supported by a private gift from Mr. Sanford and to a smaller extent by the NSF. The Homestake mine is now known as the Sanford Laboratory. It lies far from the oceans and has the deepest reaching tunnels in North America $(8000 \mathrm{ft}$ ) which provides an optimal stage for monitoring the seismic noise with a three dimensional network of seismometers.

In August of 2008 a first series of seismic measurements were performed on various underground levels in the Homestake mine. The goal was to familiarize with the working procedures in a mine, to learn how to set up an experiment in underground conditions and to obtain first results from a small array of seismometers. Three different levels were checked: the $300 \mathrm{ft}$, the $2000 \mathrm{ft}$ and $800 \mathrm{ft}$ levels. Data was collected from a Streckeisen@ STS-2 and two Trillium (C) 240T. This experiment was intended as a starting point for a more exhaustive survey. ${ }^{1}$

In December 2008, two new prototypes of monolithic seismometers, developed by the group of Applied Physics of the University of Salerno, were installed at Homestake to perform a long term test of the new seismometers aimed to characterize the seismic noise at the Homestake site in the frequency band $10^{-4} \div 30 \mathrm{~Hz}$ and to

Send correspondence to Fabrizio Barone - e-mail: fabrizio.barone@na.infn.it

Remote Sensing for Environmental Monitoring, GIS Applications, and Geology IX, edited by Ulrich Michel, Daniel L. Civco, Proc. of SPIE Vol. 7478, 74782K · (c) 2009 SPIE · CCC code: 0277-786X/09/\$18 · doi: 10.1117/12.830453 
estimate the level of Newtonian noise, both necessary to understand the feasibility of underground gravitational wave interferometers sensitive at $1 \mathrm{~Hz}$ and below. ${ }^{1}$

In the paper the tunable monolithic seismic sensor prototype, the infrastructure of the laboratory, the sensors installation at $2000 \mathrm{ft}$ and the architecture of the DAQ system are described. Then, the first scientific data acquired are presented and discussed, together with the future developments and improvements.

\section{THE MONOLITHIC SENSOR}

The instrument is basically a monolithic tunable folded pendulum, shaped with precision machining and electricdischarge-machining, that can be used both as seismometer and, in a force-feedback configuration, as accelerometer. It is a very compact instrument, very sensitive in the low-frequency seismic noise band $\left(10^{-5} \div 10 \mathrm{~Hz}\right)$, with a very good immunity to environmental noises. Tune ability of the resonance frequency and an integrated laser optical readout, consisting at this stage of an optical lever, are its main characteristics. The introduction of the interferometric readout will has largely improved the sensitivity of this version of the seismometer, ${ }^{3}$ that is now $\approx 10^{-12} \mathrm{~m} / \sqrt{\mathrm{Hz}}$ in the band $10^{-1} \div 10 \mathrm{~Hz}$.

The theoretical FP transfer function, describing the FP dynamics as seismometer and/or accelerometer, can be easily obtained using the simplified Lagrangian model developed by J.Liu et al. ${ }^{2}$ This model, based on the mechanical scheme shown in Figure 1, describes only the FP basic dynamics, but it is anyway very useful to understand the FP main characteristics and expected performances.

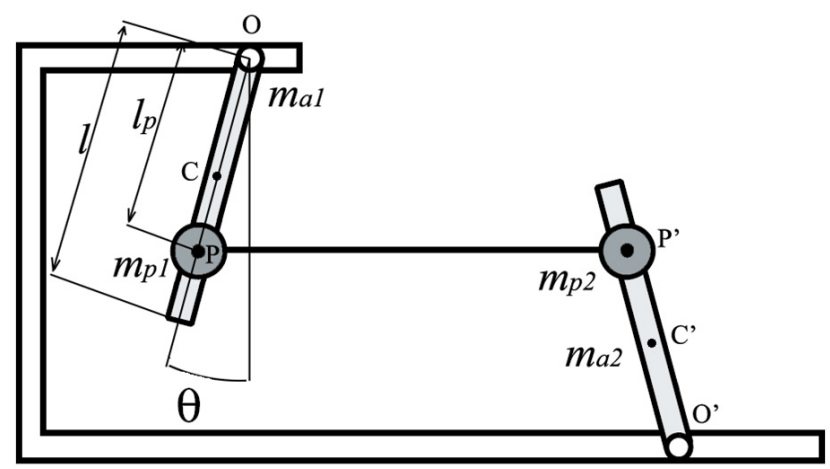

Figure 1. Folded Pendulum Mechanical Scheme

Defining the coordinate of the pendulum frame (fixed to the ground) as $x_{g}$ and the coordinate of the FP central mass as $x_{c}$ (see Figure 1), then the FP transfer function is

$$
\frac{x_{c}(\omega)}{x_{g}(\omega)}=\frac{\omega_{o}^{2}-A_{c} \omega^{2}}{\omega_{o}^{2}-\omega^{2}}=1+\frac{\left(1-A_{c}\right) \omega^{2}}{\omega_{o}^{2}-\omega^{2}}
$$

where $A_{c}$ is the parameter related to the center of percussion effects ${ }^{2}$ and

$$
\omega_{o}=\sqrt{\frac{\left(m_{a 1}-m_{a 2}\right) \frac{g l}{2 l_{p}^{2}}+\left(m_{p 1}-m_{p 2}\right) \frac{g}{l_{p}}+\frac{k_{\theta}}{l_{p}^{2}}}{\left(m_{a 1}+m_{a 2}\right) \frac{l^{2}}{3 l_{p}^{2}}+\left(m_{p 1}+m_{p 2}\right)}}
$$

is the angular resonant frequency of the FP. Finally, considering also the dissipative effects, the mass displacement transfer function with respect to the ground displacement can be obtained as

$$
\frac{x_{c}(s)-x_{g}(s)}{x_{g}(s)}=\frac{\left(A_{c}-1\right) s^{2}}{s^{2}+\frac{\omega_{o}}{Q\left(\omega_{o}\right)} s+\omega_{o}^{2}}
$$

where $Q\left(\omega_{o}\right)$ is the global quality factor. 


\subsection{The Prototype}

The FP mechanical prototype is a monolithic system, shaped with precision machining and electric discharge machining $(\mathrm{EDM}){ }^{3,4}$ In fact, the monolithic mechanical design has the great advantage of avoiding the shear effects at the contact surface among mechanical parts that can generate hysteresis and dissipation in a non monolithic structure. ${ }^{2}$ The result is a very compact sensor, with a high Q-factor (the Q factor of the material) and a good thermal sensitivity that guarantee a very good sensor directivity: coupling factors of less than $10^{-4}$ among the different degrees of freedom have been obtained in monolithic structures. ${ }^{5}$

All the tests presented in this paper were performed using an Aluminum prototype (mod. 08F_100_AL1). In fact, Aluminum $(\mathrm{Al})$ and Copper-Beryllium $(\mathrm{CuBe})$ seem to be the best materials for the implementation of a monolithic FP sensor due to their good thermal conductivity, immunity to electromagnetic fields, good elastic properties and low internal friction characteristics. We chose Aluminum for these tests simply because it is cheaper and easier to machining, and, therefore, ideal for the implementation of FP prototypes.

The monolithic sensor, shown in Figure 2 with the interferometric read out, was machined starting from a $134 \times 134 \times 40 \mathrm{~mm}$ block of metal (Alloy 7075 -T6). A thin $250 \mu \mathrm{m}$ wire cut is used to separate the pendulum arm, the inverted pendulum arm and the central mass from the frame. The four torsional flexures, connecting the pendulum arms to the central mass and to the frame, have an elliptical profile with $100 \mu \mathrm{m}$ minimum thickness with ellipticity ratio of $\epsilon=16 / 5$. The pendula arms $(71.5 \mathrm{~mm}$ length and spaced by $102 \mathrm{~mm})$ are designed to minimize the mass and the moment of inertia without reducing rigidity and symmetry. The values of the masses of the pendulum arm, of the inverted pendulum arm and of the central mass are $m_{a_{1}} \approx 40 \mathrm{~g}, m_{a_{2}} \approx 50 \mathrm{~g}$ and $\left(m_{p_{1}}+m_{p_{2}}\right) \approx 600 \mathrm{~g}$, respectively.

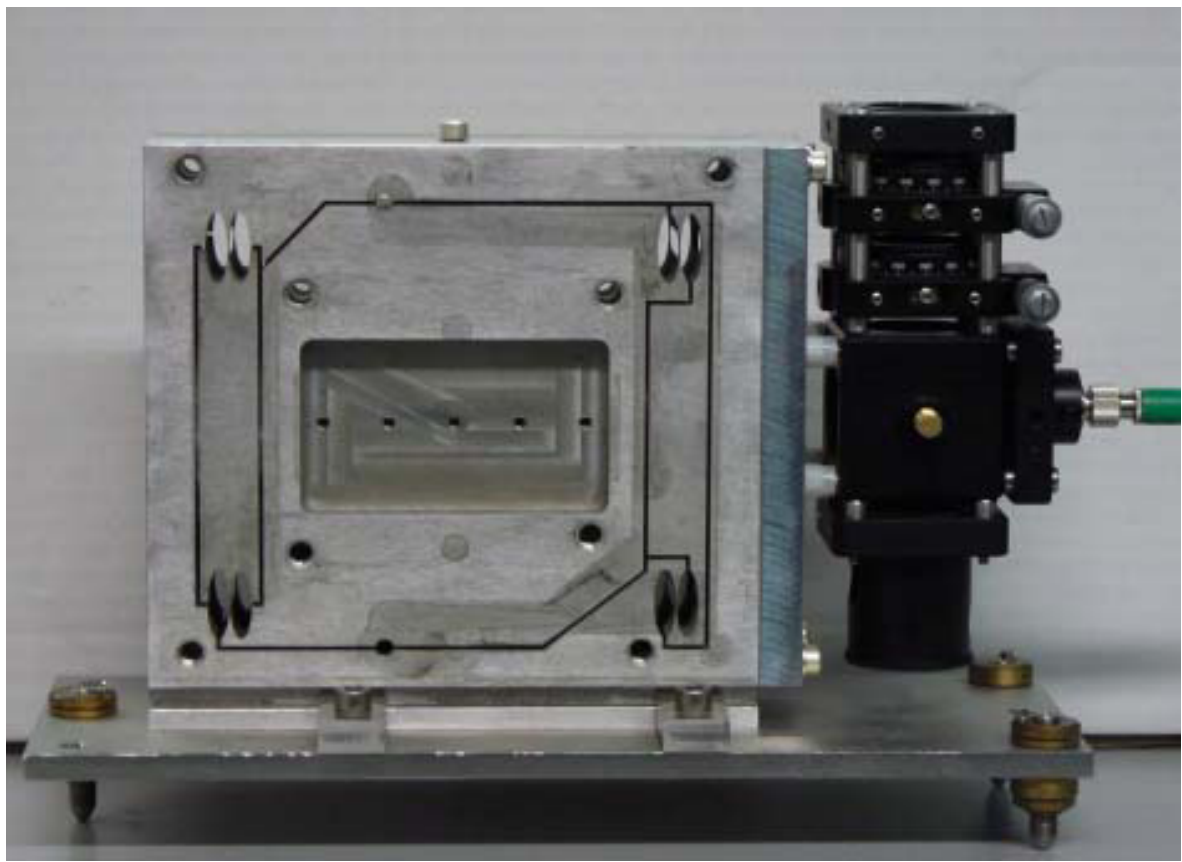

Figure 2. Picture of the horizontal monolithic Folded Pendulum sensor. On the sensor right side there is the inverted pendulum and the mirror for the optical read-out system mounted on the central mass. On the sensor left side there is the pendulum and a recess where is placed the coil-magnet actuation system for the implementation of the FP control when the sensor is used as an accelerometer. The tuning masses are placed on the central mass. On the right the optical readout (optical lever and homodyne Michelson interferometer) is shown.

The elliptic shape of the four flex joints, the monolithic FP sensor most critical part, ensure robustness and long-term durability to the mechanics, reducing the principal stress. In fact, elliptic hinges allow low values of the hinge thickness (less than $100 \mu \mathrm{m}$ ), still remaining very far from the $550 \mathrm{MPa}$ of the Alloy 7075-T6 elastic limit. Another great advantage of the elliptic hinges is that the eigenfrequency decreases at the increasing of the 
hinge ellipticity. ${ }^{6}$ A numerical simulation of the relations of the hinge ellipticity on the FP resonance frequency has been performed with finite element analysis techniques. ${ }^{3}$

The tunability of the monolithic FP natural frequency was obtained machining a large recess in the central mass to hosting suitable shaped and positioned tuning masses. In fact, tuning the FP at its lowest possible natural frequency maximizes the sensor measurement band at low frequencies. But, the lower is the natural resonance frequency, the lower is the restoring force of the pendulum to external perturbations. The drawback of soft restoring forces is that the central mass (test mass) easily touches the frame, saturating the sensor. Therefore, the gaps between the central mass-arms and arms-frame was made of $1 \mathrm{~mm}$ large. In this way we increased the dynamics of the monolithic FP sensor, still keeping it far from the elastic limit of the material. These large gaps have another advantage, very important for FP applications in air. In fact, the Q-factor of the instrument in air is strongly influenced by the damping effect of the air present in these gaps, that largely reduces the best possible value, that is $Q \approx 3000$ of the Aluminum in vacuum. Values of $Q$ as low as $Q=3$ were measured for applications of the FP sensor in air. ${ }^{5}$ Our technical choice allowed us to obtaining a measured value of $Q=140$ in air at $f_{o}=0.720 \pm 0.005 \mathrm{mHz}$, perfectly acceptable for an use as sensor in air.

The configuration of the monolithic FP as seismometer is straightforward. In fact, as shown before, the readout output signal $\left(x_{p}(\omega)-x_{s}(\omega)\right)$ is acquired, calibrated and then processed according to Equation 3 . We measured the three sensitivity curves corresponding to optical levers (with PSD and quadrant photodiodes) and interferometer readouts. In Figure 3 the best theoretical and experimental sensitivities curves at $T=300 \mathrm{~K}$ and $f_{o}=70 \mathrm{mHz}$ in air and without thermal stabilization are shown.

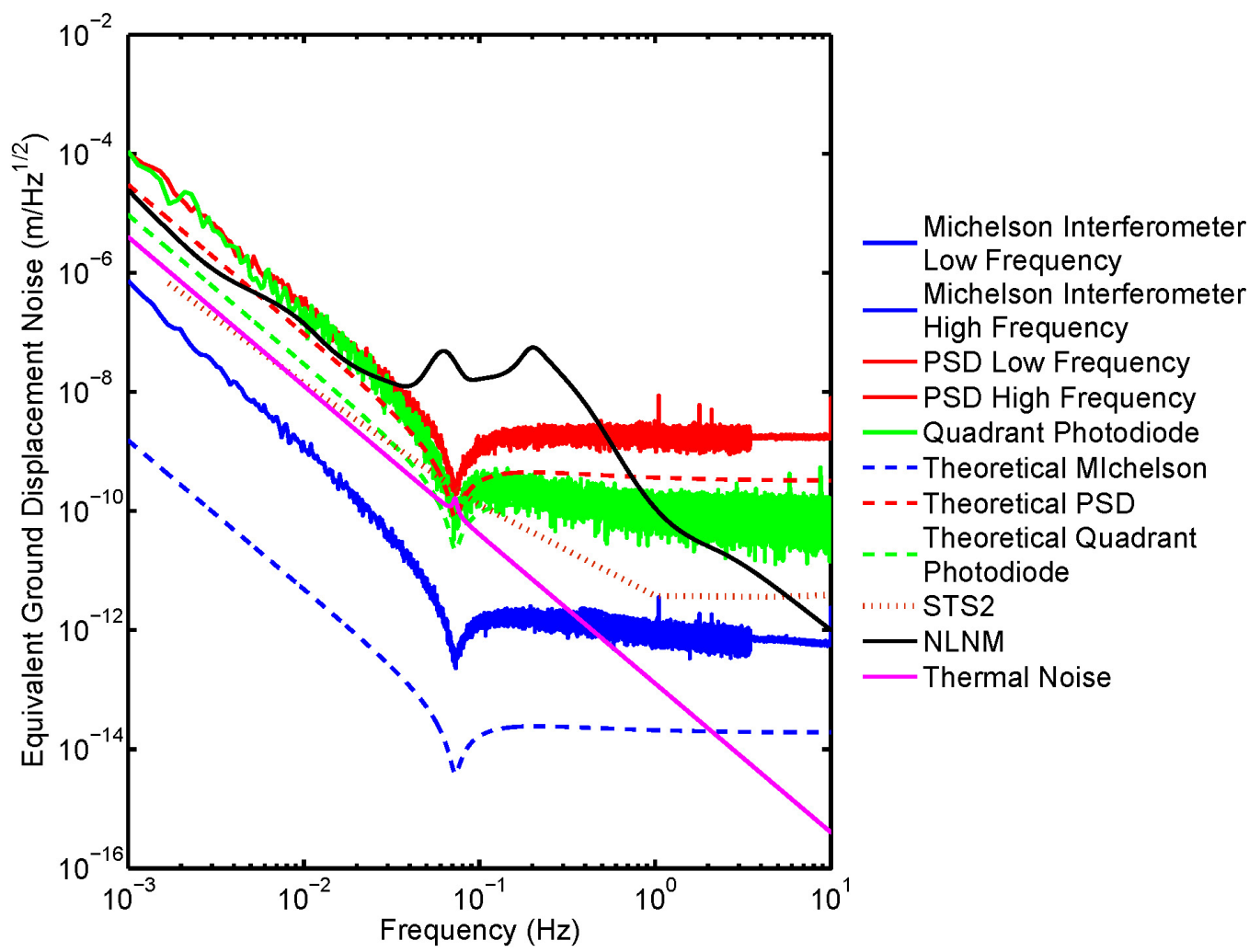

Figure 3. Theoretical and experimental sensitivity curves of the monolithic FP seismometer with optical lever and laser interferometric readouts. Note that the theoretical curves were evaluated at $T=300 \mathrm{~K}$, while the best experimental sensitivities were evaluated for a seismometer with the best obtained natural frequency $f_{o}=70 \mathrm{mHz}$ in air and without thermal stabilization.

To compare the sensitivity of the FP seismometer with other geophysical instruments, we reported in Figure 3 also the sensitivity of the STS-2 by Streckeisen, that represents the state-of-art of the low frequency seismic sensors. ${ }^{7}$ This Figure shows that the monolithic FP sensor has already a sensitivity comparable or better (with 
interferometric readout) of the STS-2 in the band $10^{-3} \div 10 \mathrm{~Hz}$. We reported also the Peterson New Low Noise Model (NLNM), ${ }^{8}$ that is the Minimum Earth Noise evaluated from a collection of seismic data from 75 sites located around the world, that describes the minimum level of earth noise: noise levels below this are never - or extremely rarely - observed.

\section{THE SEISMIC STATIONS}

The $2000 \mathrm{ft}$ site was built in August 2008, organised as a favourite station inside the mine for a long time. It was built on the concrete platform of an old charging station located on the bedrock, having carefully checked that any audible source of dropping water were eliminated as good as possible. The $2000 \mathrm{ft}$ level station was obtained sectioning a tunnel and sealing it from two sides with double layered walls. The instruments were located in the station inside a specially designed insulated box. A separate hut at some distance to the instrument area hosts the data acquisition PCs and the DC power supply. ${ }^{1}$

The environmental conditions at the $2000 \mathrm{ft}$ level are stable around $20^{\circ} \mathrm{C}$ with an rms of about $0.2^{\circ} \mathrm{C}$. Sensors are installed close to the seismometers to monitor the most important environmental variables: temperature, pressure, humidity, sound and magnetic field. One of the most important goals of the environmental monitoring is to send a warning if the humidity levels approach $100 \%$ and condensation of water on the electronics could start soon. Is important to underline that these humidity sensors operate in highly humid environments (90\%). In any case, the insulation box are good enough to bring humidity down to $80 \%-90 \%$ also in presence of $100 \%$ humidity. It is also important to underline the importance of pressure and sound monitoring. In fact, pressure and sound fluctuations may show significant correlation with seismic data since both could generate rock vibrations or may even directly act on seismometers despite all efforts to isolate the instruments. These sensors are very important as soon as one tries to understand a seismic spectrum in detail.

The data-acquisition system includes the configuration of the local network and remote access to PCs. The acquisition system is based on the PCI 6289 card from National Instruments@. It contains a 18 bit ADC and an internal amplifier with a maximum amplification of 100 . The input range is limited to $\pm 10 \mathrm{~V}$. The Data Acquisition is managed by Labview(C) programs which also generate analog-output signals to initiate masscentering procedures of seismometers if necessary. The data is first stored in ASCII files as $128 s$ records sampled at $100 \mathrm{~Hz}$. Then the data is made available to the outside world by an ftp server while the timing signals are provided to the acquisition system through optical fibers. The sequence of installation of the monolithic sensor within the insulated box of the $2000 \mathrm{ft}$ station is shown Figure 4. A twin Data Acquisition station with two FP monolithic sensors is located at the University of Salerno in continuous monitoring. Its purpose is that of perfoming long term monitoring to optimize the performance of the sensors and to reproduce the situations and the errors that may happen in the $2000 \mathrm{ft}$ station in DUSEL, to remotely solve the problems and, at the same time, to improve the instruments.
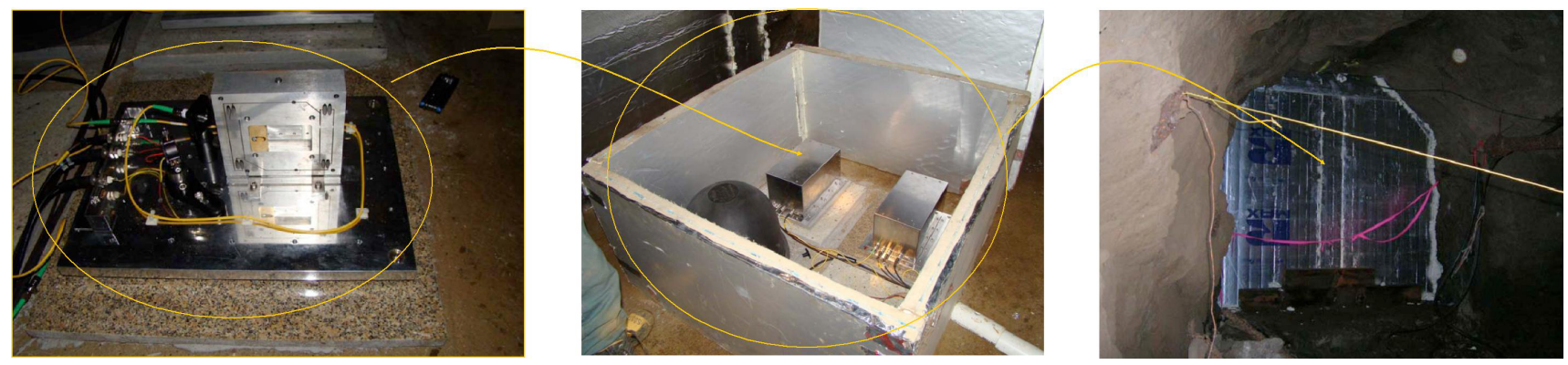

Figure 4. Sequence of the installation of the monolithic sensor prototype within the insulated box of the $2000 \mathrm{ft}$ station

\section{EXPERIMENTAL RESULTS}

In this section we present some of the preliminary results of the first month of data acquisition of FP monolithic sensor prototype. In particular, the power spectral density of ground displacment at $2000 \mathrm{ft}$ underground level 
(compared with the theoretical expected FP noise and Peterson's New Low Noise Model) is shown in Figure 5. What is relevant is the large measurement band $\left(10^{-} 5 \div 30 \mathrm{~Hz}\right)$ and small dimensions of the sensor coupled with a good sensitivity, although the latter is largely limited by our choice of using an optical lever as readout for these first tests. Of course, the apparent limitation in the low frequency band is simply due to the fact that the power spectral density has been evaluated only for one month data. Longest runs will allow the exploration of lower frequency regions. In fact, the FP monolithic sensor is an open loop sensor, so that there are no limitations coming from the feed-back control, as it happens in the majority of the commercial instruments used for seismic noise acquisition.

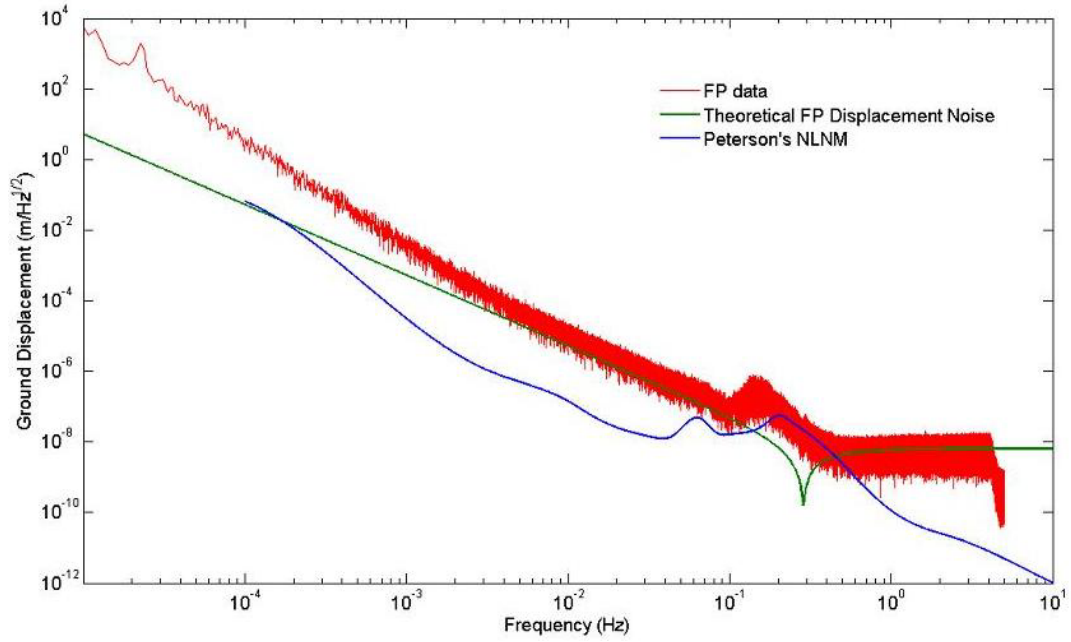

Figure 5. Power Spectral Density of ground displacement at $2000 \mathrm{ft}$ underground level (compared with theoretical FP noise and Peterson's New Low Noise Model)

It is worth underlining that the limitations in sensitivity are mainly due to the safe resonance frequency chosen for this first test in the Homestake mine $(300 \mathrm{mHz})$ and to the optical lever chosen as optical readout system, instead of the more sensitive interferometric readout. In particular, tuning the FP sensor at a lower resonance frequency would simply translate the sensitivity curve towards the low frequency region, enlarging the low frequency band. Nevertheless, even with this not optimal configuration, the instruments is able to detect part of the Peterson Low Noise Spectrum and, in the very low frequency region, the peaks due to Earth tides (Figure 5).
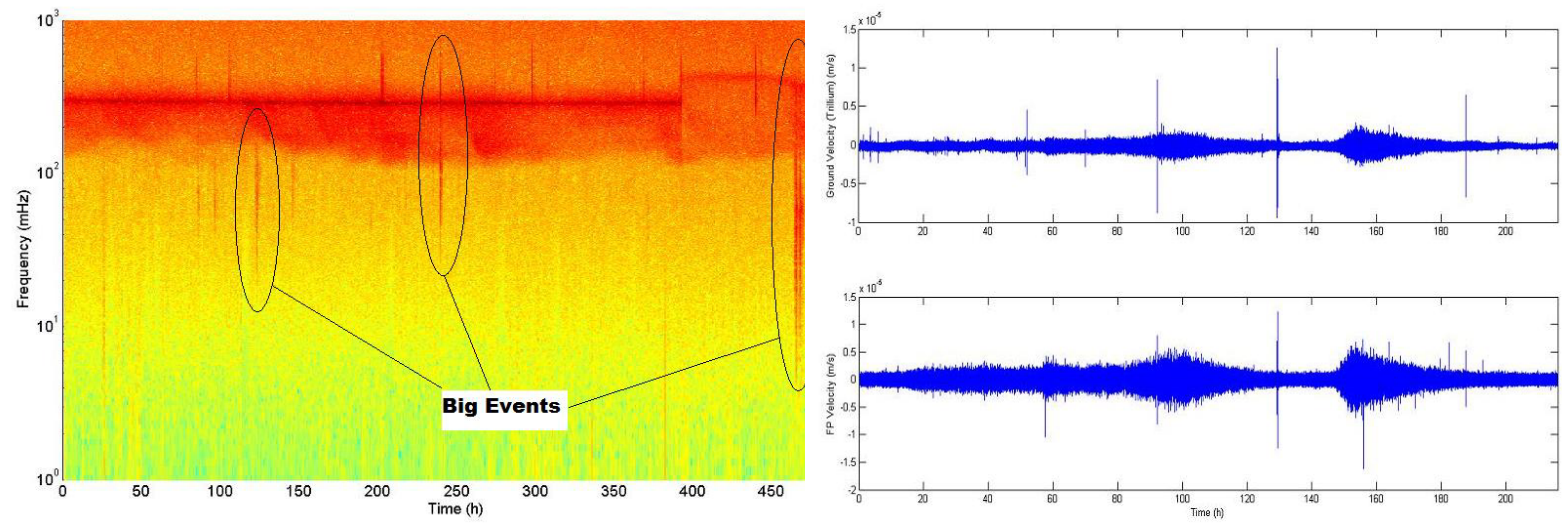

Figure 6. On the left spectrogram of $\mathrm{FP}$ velocity data of $500 h$; on the right ground velocity measurement comparison between the ground velocity measurement by Trillium240@ (Nanometrics) (up) and by our seismometer (down)

For completeness, in Figure 6 the spectrogram of FP velocity of $500 h$ data and the ground velocity measurement comparison between the ground velocity measurement by Trillium240(C) (Nanometrics) and by our 
seismometer are shown.

Finally in Figure 7 an example of teleseismic earthquake recorded by the monolithic FP seismometer with a band pass filter between $1 \mu \mathrm{Hz}$ and $10 \mathrm{mHz}$ is shown.

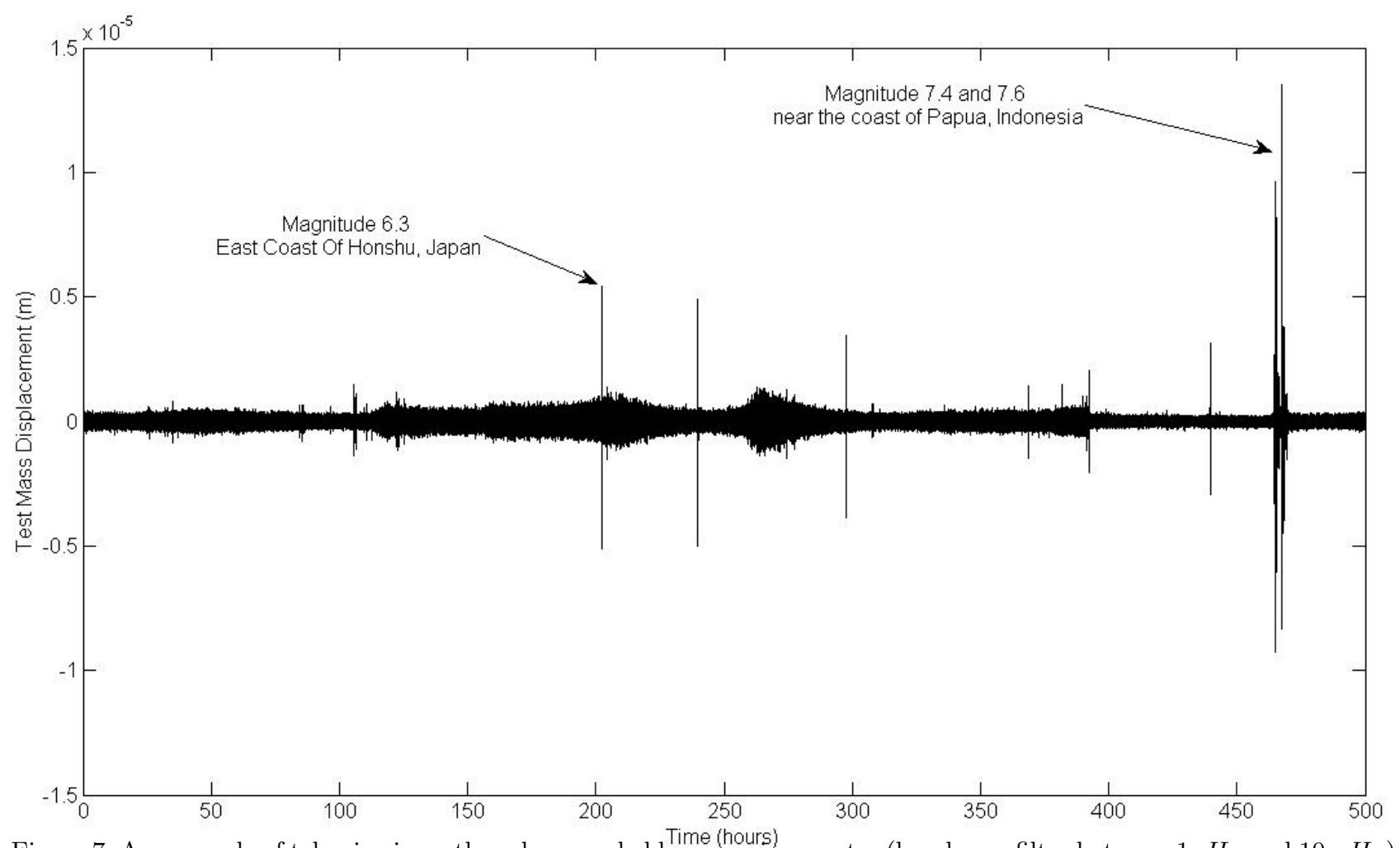

Figure 7. An example of teleseismic earthquake recorded by our selsmometer (band pass filter between $1 \mu H z$ and $10 \mathrm{mHz}$ ).

\section{CONCLUSIONS AND FUTURE DEVELOPMENTS}

Two FP monolithic sensors are installed into the $2000 \mathrm{ft}$ station in the Homestake mine and are fully operational. This station is provided with a Power Line with UPS, a National Instruments (c) (32 channels - 18 bit ADC) data acquisition system, a network access through optical fibre cables and an environmental monitoring system. Other sensor, like a Nanometrics T240 seismometer and a Streckeisen STS-2 are installed in the same station. The FP sensor sensitivity is very close to the theoretical one for a resonance frequency of about $300 \mathrm{mHz}$. Although better sensitivities can be easily obtained reducing the resonance frequency of the instrument using the tuning mass (down to less than $100 \mathrm{mHz}$ ), it was preferred to privilege instrument robustness for this first unattended measurement in the Homestake mine.

Relevant is the large measurement band of this sensor $\left(10^{-} 5 \div 30 \mathrm{~Hz}\right)$ coupled with a good sensitivity and small dimensions, although largely limited by our choice of using an optical lever as readout for these first tests. The limitation in the low frequency band is simply due to the fact that the power spectral density has been evaluated only for one month data. Longest runs will allow the exploration of lower frequency region. Nevertheless, even with this not optimal configuration, the instruments is able to detect part of the Peterson Low Noise Spectrum and, in the very low frequency region, the peaks due to Earth tides.

Improvements of the monolithic sensors are scheduled before the end of the year: a reduction of the resonance frequency and a replacement of the optical readout and conditioning electronics to improve its performances at low frequency. Further sensors will be located in a new station under construction located at $4100 \mathrm{ft}$, to analyze and cross correlate the seismic noise at different depths. 


\section{ACKNOWLEDGMENTS}

We acknowledge Galli \& Morelli Factory and, in particular, Mr. Carlo Galli for the useful suggestions and for the technical support in the development and implementation of mechanical part of the monolithic FP sensor.

This work is part of the PRIN 2007 Research Project Low frequency sensitivity in underground cryogenic gravitational wave interferometers (Project 2007NXMBHP), partly supported by the Ministero dell'Istruzione, dell'Università e della Ricerca (MIUR), Italy.

\section{REFERENCES}

[1] F. Acernese, R. DeSalvo, J. Harms, A. Sajeva, L. Shaffner, T. Trancynger, Seismic stations implementation and improvements at Homestake, LIGO T080344-00-R, Caltech, USA (2008).

[2] J. Liu, L. Ju, D.G. Blair, Phys. Lett. A, 228, 243-249 (1997).

[3] F. Acernese, R. De Rosa, G. Giordano, R. Romano, F. Barone, Rev. Sci. Instrum., 79, 074501, doi:10.1063/1.2943415 (2008).

[4] F. Acernese, R. De Rosa, G. Giordano, R. Romano, F. Barone, Tunable mechanical monolithic horizontal accelerometer for low frequency seismic noise measurement in Sensors and Smart Structures Technologies for Civil, Mechanical, and Aerospace Systems 2009, edited by Masayoshi Tomizuka, Chung-Bang Yun, Victor Goirgiutu, Proceedings of SPIE Vol. 7292, SPIE, Bellingham, WA, 69320K, 72922J, doi: 10.1117/12.814106 (2009).

[5] A. Bertolini et al., Nucl. Instr. and Meth., 556, 616-623 (2006).

[6] M. Y. Tseytlin, Rev. Sci. Instrument, 73, 3363 (2002).

[7] Y. Nakayama et al., Performances test of STS-2 seismometers with various data loggers, in proceedings of IWAA2004, CERN, Geneva, 4-7 October 2004.

[8] J. Berger, P. Davis, Ambient Earth Noise: A Survey of the Global Seismographic Network, in 2005 IRIS 5-Year Proposal, 38 (2005). 